\title{
Interactive comment on "Assessing Green and Blue Water: Understanding Interactions and Making Balance between Human and Nature" by Ganquan Mao et al.
}

\section{S. Mylevaganam}

mylevaganamsivarajah@gmail.com

Received and published: 7 May 2018

1) In the current version of the manuscript, the distinction between semi-distributed and fully distributed hydrological models is not apparent. What is meant by semi-distributed hydrological model? What is meant by fully distributed hydrological model? On what basis the selected model is considered as a fully distributed hydrological model (see P-4 LN-28)? Is the definition of fully distributed model meant for simulating the "necessary" hydrological element for the analysis of interest (see P-4 LN-31)? If the definition of fully distributed model is preserved only for the "necessary" elements, how would it lead to conclude that the selected model is a fully distributed hydrological model? 
Are there elements other than the necessary elements that are not fully described in the selected model? From the reader's point of view, having a fully distributed hydrological model is precluded with our understanding on the processes that define the hydrological system, even though many efforts have been made and are in progress to better understand the hydrological system. Moreover, from the reader's point of view, in no way, the selected model can represent a fully distributed hydrological model, considering the way the selected model has been conceptualized as described in the manuscript(see P-5 LN-1-15).

2) The current version of the manuscript is based on a model that was calibrated and validated by Tian et al, 2015(https://www.sciencedirect.com/science/article/pii/S1364815214003016). Therefore, the research work that is carried out and presented in the current version of the manuscript solely depends on the calibrated and validated model. Moreover, around $82.1 \%$ of the LULC (see P-4 LN-14) of the domain of interest is desert. Therefore, it becomes paramount to evaluate the calibrated and validated model. Otherwise, the evaluation of this manuscript will be based on the assumption that the authors of that manuscript (https://www.sciencedirect.com/science/article/pii/S1364815214003016) are reliable and well-known in the field of hydrology, and/or the editorial board went through the calibrated and validated model carefully and ensured that the model is flawless. Moreover, the published manuscript (https://www.sciencedirect.com/science/article/pii/S1364815214003016)) is not feely available for a reader of an open-access journal (i.e., HESS) to evaluate this manuscript.

3) As per the "new" framework (see P-1 LN-11) implemented by the authors, the green and blue water resources are calculated for each pixel and then summed up (see P-6 $\mathrm{LN}-11$ ). What is meant by this framework (fully distributed? new framework?)? How did the authors implement the streamflow routing? How did the authors account the pixels that represent the stream network? How did the authors calculate the surface

Printer-friendly version

Discussion paper 
runoff/excess rainfall at the uppermost point of a reach, which got routed along the reach? Moreover, what is the meaning of green water in the deserts (i.e., $82.1 \%$ of LULC)? Are these green water available in the root system for the plants in the deserts? If this is the case, the classification of LULC is misleading? Why are those areas considered as deserts?

4) As per the authors, the study area (i.e., HRB) is impacted by "heavy human activities" and the hydrological cycle is "dramatically" altered. Moreover, the study area has "strong" GW and SW exchanges (see P-4 LN-5). Do these statements need supportive texts/references?

Interactive comment on Hydrol. Earth Syst. Sci. Discuss., https://doi.org/10.5194/hess-2018193, 2018. 